\title{
Clinical utility of imaging for evaluation of hepatocellular carcinoma
}

\author{
This article was published in the following Dove Press journal: \\ Journal of Hepatocellular Carcinoma \\ 14 July 2014 \\ Number of times this article has been viewed
}

\author{
Takamichi Murakami' \\ Masakatsu Tsurusaki' \\ Tomoko Hyodo' \\ Yasuharu Imai ${ }^{2}$ \\ 'Department of Radiology, Kinki \\ University Faculty of Medicine, \\ ${ }^{2}$ Department of Hepatology and \\ Gastroenterology, Ikeda Municipal \\ Hospital, Osaka, Japan
}

Correspondence: Takamichi Murakami Department of Radiology, Kinki University Faculty of Medicine, 377-2 Ohno-Higashi, Osaka-Sayama, Osaka 589-85II, Japan

Tel +8I7 2366022 I

Fax +81723671685

Email murakami@med.kindai.ac.jp

\begin{abstract}
The hemodynamics of a hepatocellular nodule is the most important imaging parameter used to characterize various hepatocellular nodules in liver cirrhosis, because sequential changes occur in the feeding vessels and hemodynamic status during hepatocarcinogenesis. Therefore, the imaging criteria for hepatocellular carcinoma (HCC) are also usually based on vascular findings, eg, early arterial uptake followed by washout in the portal venous and equilibrium phases. Contrast-enhanced ultrasonography, dynamic multidetector-row computed tomography (MDCT), and dynamic magnetic resonance (MR) imaging with gadopentetate dimeglumine (GdDTPA) are useful for detecting hypervascular HCC on the basis of vascular criteria but are not as useful for hypovascular HCC. Contrast-enhanced MR imaging with gadolinium ethoxybenzyl diethylenetriamine pentaacetic acid (Gd-EOB-DTPA), a hepatocyte-specific MR contrast agent, is superior to dynamic MDCT and dynamic MR imaging with Gd-DTPA in detecting both hypervascular and hypovascular HCC. Moreover, Gd-EOB-DTPA-enhanced MR imaging can display each histologically differentiated HCC as hypointense relative to the liver parenchyma. ${ }^{18} \mathrm{~F}$-fluorodeoxyglucose positron emission tomography imaging might not be suitable for the screening and detection of HCC, given its lower diagnostic performance. However, this technique plays an important role in determining whether HCC has spread beyond the liver.
\end{abstract}

Keywords: hepatocellular carcinoma, evaluation, imaging, clinical utility

\section{Introduction}

The annual carcinogenesis rates for liver cirrhosis related to hepatitis $\mathrm{B}$ and $\mathrm{C}$ are $2.5 \%-3 \%$ and $5 \%-8 \%$, respectively, and such cases are classified as very high risk. High-risk factors that are thought to increase the risk of carcinogenesis include male sex, older age, alcohol use, obesity, diabetes, high alpha-fetoprotein levels, and a low platelet number. ${ }^{1,2}$ In patients with hepatitis $\mathrm{C}$, the progression of liver fibrosis increases the carcinogenic risk. The progression of liver fibrosis is related to a decrease in the platelet count, so a platelet count $<100,000 \mathrm{~mm}^{2}$ is another risk factor. The rate of carcinogenesis increases dramatically in older patients with hepatitis C. On the other hand, in hepatitis B, carcinogenesis can be observed early after infection and in young patients. ${ }^{1,2}$

Recently, the incidence of carcinogenesis has increased in non-B, non- $C$ hepatitis patients. Nearly all of these patients suffer from alcoholic hepatitis, autoimmune hepatitis, or nonalcoholic steatohepatitis; however, some have no basal liver disease. Carcinogenesis associated with alcoholic hepatitis, autoimmune hepatitis, or primary biliary cirrhosis is related to liver cirrhosis with progressive fibrosis. ${ }^{1,2}$

For the surveillance and early detection of hepatocellular carcinoma (HCC), tumor markers such as alpha-fetoprotein, AFP-L3 (lens culinaris agglutinin-reactive 
fraction of alpha-fetoprotein), PIVKA-II (protein induced by vitamin $\mathrm{K}$ absence or antagonist-II), and imaging modalities are usually employed. Ultrasonography (US) is the firstchoice imaging modality for HCC screening because of its low cost, minimally invasive nature, and convenience. Dynamic computed tomography (CT) or dynamic magnetic resonance (MR) imaging are recommended for the screening of patients with suspected early-stage HCC or high-risk patients with liver cirrhosis. However, recently, gadolinium ethoxybenzyl diethylenetriamine pentaacetic acid (Gd-EOB-DTPA)-enhanced MR imaging is being increasingly recommended. Positron emission tomography (PET) is widely available, and its oncologic applications with the 2-[18F] fluoro-2-deoxy-D-glucose $\left({ }^{18} \mathrm{~F}-\mathrm{FDG}\right)$ tracer have become a standard imaging modality in tumor diagnosis and staging; however, its sensitivity for HCC is not high. In this review, we discuss the clinical utility of these imaging modalities for HCC.

\section{Imaging modalities}

Less invasive imaging modalities, such as US, CT, and MR imaging, have evolved considerably in recent times, and are essential for imaging-based screening of HCC.

\section{Ultrasound}

Gray-scale US is very important and useful for the screening or surveillance of $\mathrm{HCC}^{3}$ and is sometimes useful for the differential diagnosis of liver tumors, particularly those with characteristic findings. US is inexpensive and noninvasive. However, it is sometimes difficult to precisely evaluate the whole liver by US in patients with obesity or liver atrophy and in those who cannot hold their breath.

The specific US findings of HCC are mosaic pattern, peripheral halo sign, and posterior enhancement. An evaluation of the arterial flow by Doppler US is also useful for diagnosing hypervascular (typical) HCC. However, this is difficult in small HCC because early-stage tumors are usually hypovascular. In addition, well differentiated HCC might have high echogenicity, reflecting intratumoral fat deposition (fatty metamorphosis) and thus might be difficult to differentiate from hemangioma, which also has high echogenicity. Conventional color Doppler US and power Doppler US have a limited ability to depict intralesional vascularity because both techniques are insensitive to slowflowing and deeply located blood vessels, and are usually associated with multiple artifacts. Therefore, the diagnostic capabilities of these techniques for HCC are limited. For such lesions, contrast-enhanced US, dynamic CT, or MR imaging should be performed for the differential diagnosis.

Contrast-enhanced US is helpful for correctly diagnosing HCC. A variety of microbubble-based contrast agents for liver US are currently available for clinical use in many European, Asian, and South American countries, ${ }^{4}$ eg, SonoVue ${ }^{\circledR}$ (Bracco Imaging, Milan, Italy), Definity ${ }^{\circledR}$ (Lantheus Medical Imaging, Boston, MA, USA), and Sonazoid ${ }^{\circledR}$ (Daiichi Sankyo, Tokyo, Japan). These contrast media facilitate the evaluation of liver tumor hemodynamics, including the hypervascularity (hyperenhancement in the arterial phase) associated with typical HCC. Contrast-enhanced US is most sensitive for evaluating the arterial enhancement of HCC.

Negative enhancement or "washout of contrast medium" during the portal venous and equilibrium phases is another important characteristic of HCC, as typical lesions lack a portal venous supply. In HCC, washout is generally slower and milder than in liver metastasis. ${ }^{5}$

Benign nodules, such as regenerative or dysplastic nodules, are usually isoechoic or slightly hypoechoic in the arterial and portal venous phases. Contrast-enhanced US is helpful for characterizing potential mimickers of HCC on imaging, such as nontumoral arterioportal shunts or hemangiomas.

Sonazoid can evaluate not only the tissue vascularity but also the presence of Kupffer cells because it accumulates in these cells in the Kupffer (post-vascular) phase. ${ }^{6}$ Typical hypervascular HCCs usually lack Kupffer cells, whereas benign hepatocellular lesions contain these cells. Therefore, Sonazoid is useful for differential diagnosis. However, its diagnostic utility for early-stage HCC and well differentiated $\mathrm{HCC}$ has not been confirmed because these tumors often contain Kupffer cells.

Sonazoid-enhanced US detection of focal liver lesions has been reported to be superior to that of multidetector row computed tomography (MDCT). ${ }^{7}$ Contrast-enhanced US is now recognized as a useful imaging modality for the noninvasive diagnosis of newly detected small liver nodules during HCC surveillance. ${ }^{8}$ The sensitivity, specificity, and positive predictive value of contrast-enhanced US for diagnosis of $\mathrm{HCC}$ is $88.8 \%, 89.2 \%$, and $91.3 \%$, respectively. ${ }^{9}$ This diagnostic ability is strongly associated with nodular size; the sensitivity in nodules $1.0-2.0,2.1-3.0$, and $3.1-5.0 \mathrm{~cm}$ in size is $69 \%-80 \%, 97 \%$, and $100 \%$, respectively, and the accuracy is $82 \%-87 \%, 97 \%$, and $100 \%$. $^{10}$

\section{Computed tomography}

Dynamic CT with a bolus injection of contrast medium is essential for diagnosing liver tumors. ${ }^{11,12}$ Moreover, dynamic 
MDCT has been recently employed for liver imaging because MDCT with increased gantry rotation speeds and numbers of detector rows has become widely available. ${ }^{11,13}$

Typical HCC usually exhibits hypervascularity in the arterial phase and contrast washout in the portal venous and equilibrium phases of dynamic MDCT. These findings are useful for the diagnosis (Figure 1). The arterial phase comprises the most important set of images because, during the arterial phase, HCC displays strong contrast uptake relative to the low enhancement of the liver parenchyma. However, early HCC or borderline lesions exhibit isoattenuation to low attenuation to the liver parenchyma in the arterial phase and isoattenuation in the equilibrium phase; therefore, dynamic CT is less useful for such lesions.

Currently, low-dose CT is expected to afford a novel and less invasive examination. Iodine contrast agents are more conspicuous at low tube voltage (eg, $80 \mathrm{kVp}$ ) than at high tube voltage (120-140 kVp) settings, ${ }^{14,15}$ and hypervascular $\mathrm{HCC}$ can thus be demonstrated more clearly with low-dose CT. CT images taken at $80 \mathrm{kVp}$ might display a higher contrast-to-noise ratio for hypervascular HCCs. ${ }^{16}$ Moreover, the radiation dose is significantly reduced when CT images are obtained at $80 \mathrm{kVp}$ versus $140 \mathrm{kVp} .{ }^{17}$ Low-dose imaging might result in high levels of image noise, particularly in large patients, ${ }^{18}$ but new imaging algorithms such as iterative reconstruction can dramatically reduce the image noise and improve the image quality.
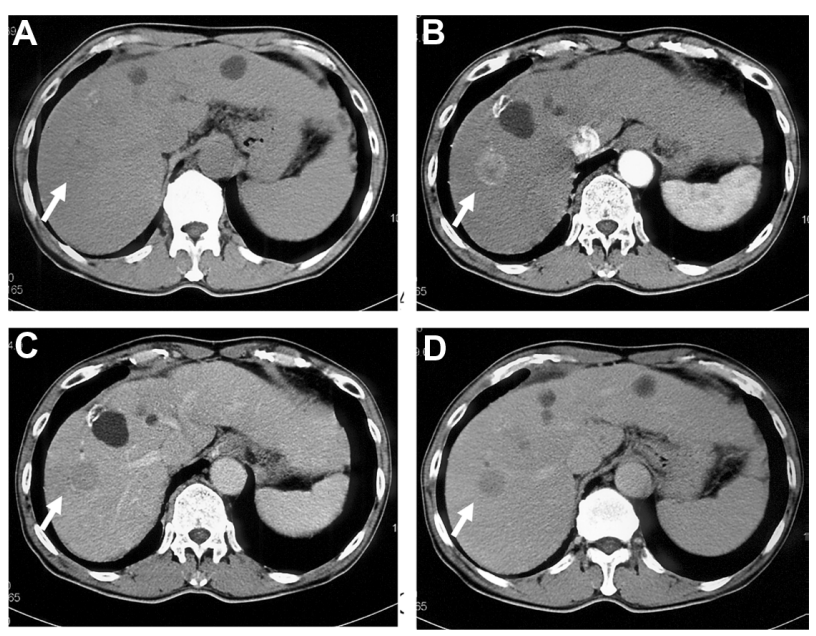

Figure I A 68-year-old man with a hepatocellular carcinoma in segment 8.

Notes: (A) A noncontrast CT image cannot clearly depict a liver nodule (arrow). (B) Arterial-phase CT image (performed with an iodine concentration of 370 $\mathrm{mg} \mathrm{I} / \mathrm{mL}$ ) shows a hyperattenuating nodular lesion in segment 8 (arrow). (C) On portal-phase CT imaging, the lesion is depicted as a fairly discrete hypoattenuating nodule (arrow). (D) An equilibrium-phase CT image shows a discrete hypoattenuating nodule (arrow). On combination of four-phase image sets, the lesion is definitely diagnosed as typical hypervascular hepatocellular carcinoma.

Abbreviation: CT, computed tomography.

\section{Magnetic resonance imaging}

Typical HCC lesions are hypointense on T1-weighted and hyperintense on T2-weighted images; in contrast, well differentiated or early HCC often exhibits hyperintensity to isointensity on T1-weighted images and isointensity to hypointensity on T2-weighted images.

Nonspecific (extracellular) contrast medium, such as gadopentetate dimeglumine (Gd-DTPA), has been used widely in dynamic MR imaging. Typical HCC exhibits hyperenhancement in the arterial phase and contrast washout in the portal venous and equilibrium phases on dynamic MR imaging, similar to the features observed with dynamic CT. In fact, the sensitivity of hypervascular HCC detection is not significantly different between dynamic MDCT and dynamic MR imaging. ${ }^{12}$ Therefore, when selecting imaging modalities, we should consider not only the sensitivity for detection of HCC but also the image quality, cost, and radiation exposure.

For MR imaging of the liver, tissue-specific MR contrast media that accumulate in Kupffer cells via phagocytosis or in hepatic cells via hepatocyte functioning are clinically available, and these media increase the tumor detection sensitivity. ${ }^{19-21}$ In particular, Gd-EOB-DTPA, a T1-shortening agent, has become an essential contrast medium for liver imaging because of its high diagnostic capacity. ${ }^{20-24}$

Gd-EOB-DTPA acts as both an extracellular and hepatocyte-specific contrast agent, enabling evaluation of the hemodynamics of a liver tumor. ${ }^{25}$ Typical HCC exhibits hyperenhancement in the arterial phase of dynamic MR imaging with Gd-EOB-DTPA and a T1-weighted sequence. Moreover, Gd-EOB-DTPA is taken up by hepatocytes in the hepatocyte phase, which occurs approximately 15-20 minutes after injection of contrast medium. However, in the absence of hepatocyte function, HCC does not take up Gd-EOB-DTPA; therefore, such HCC lesions will be hypointense relative to the liver parenchyma, which does take up Gd-EOB-DTPA. ${ }^{26,27}$ Some hypervascular and well or moderately differentiated HCC (5\%-10\%) exhibit hyperintensity in the hepatocyte phase. ${ }^{22,28}$ These nodules express OATP1B3 (OATP8) on their cell membranes, which acts as a transporter of Gd-EOB-DTPA. As such, these nodules can take up Gd-EOB-DTPA. ${ }^{29-31}$ Focal nodular hyperplasia is another type of hepatocellular nodule that exhibits hyperintensity in the hepatocyte phase. Focal nodular hyperplasia is usually isointense in the hepatocyte phase and presents with wheel-like vessels on contrast-enhanced US. These imaging findings facilitate the differential diagnosis. 
Gd-EOB-DTPA-enhanced MR imaging is superior to dynamic MDCT and dynamic MR imaging with Gd-DTPA for detection of both hypervascular and hypovascular HCC; $; 2-34$ it can detect very tiny lesions in the hepatocyte phase that cannot be detected on dynamic CT and dynamic MR imaging with Gd-DTPA. Moreover, Gd-EOB-DTPA-enhanced MR imaging can show each histologically differentiated HCC as hypointense relative to the liver parenchyma. ${ }^{22,23}$ This means that hypovascular and well differentiated HCC, including early $\mathrm{HCC}$, could appear as hypointense areas in the hepatocyte phase. ${ }^{22}$

\section{Positron emission tomography}

PET is widely available, and its oncologic application with ${ }^{18}$ F-FDG has become a standard imaging modality for diagnosis and staging of tumors. However, the sensitivity of FDG-PET for detecting $\mathrm{HCC}$ is only $50 \%,{ }^{35}$ because in the normal liver, the concentration of glucose-6-phosphatase is high, leading to rapid clearance of glucose-6-phosphate or FDG-6-phosphate from hepatocytes and a consequently mild appearance of the liver on PET. On the other hand, the detection of extrahepatic FDG-avid metastases that originated from HCC has also been reported; these metastases appear to be more FDG-avid, especially in cases of less differentiated HCC. Sugiyama et al reported that the sensitivity of FDGPET was $83 \%$ for extrahepatic metastases $>1 \mathrm{~cm}$ at the largest diameter and $13 \%$ for lesions $\leq 1 \mathrm{~cm}$. There were no false-positive lesions among the investigated. ${ }^{36}$ Therefore, the most important roles of FDG-PET for HCC patients are assessment of the malignant potential of hepatic lesions of unknown origin via simultaneous visualization of the liver and extrahepatic tissue and assessment of known HCC with clinically suspected extrahepatic metastasis.

\section{Screening}

As a diagnostic algorithm, US is the first-choice imaging modality for HCC screening. The American Association for the Study of Liver Diseases and European Association for the Study of the Liver guidelines ${ }^{3,37}$ accept only four-phase CT or dynamic contrast MR imaging as the second-choice examination modality for HCC diagnosis and contrast-enhanced US as the third choice. The Asian Pacific Association for the Study of the Liver and Japanese Society of Hepatology guidelines ${ }^{38,39}$ also accept contrast-enhanced US as a diagnostic tool. The Japanese Society of Hepatology guidelines even accept contrast-enhanced US as the second-choice modality; however, the Asian Pacific Association for the Study of the Liver guidelines recommend use of either CT or MR imaging as the second-choice modality, with contrast-enhanced US as the third-choice modality in the absence of typical diagnostic CT and/or MR imaging findings.

\section{Vascularity of HCC}

The hemodynamics represent the most important imaging finding for characterization of various hepatocellular nodules in the context of liver cirrhosis because sequential changes occur in the supplying vessels and the hemodynamic state during hepatocarcinogenesis. ${ }^{40}$ Typical HCC usually exhibits hypervascularity, whereas early and well differentiated HCC exhibits hypovascularity. Regenerative nodules and benign hyperplastic nodules usually contain normal hepatic arteries and portal veins within the lesion. ${ }^{41}$ It is very important to identify these imaging findings in each modality when diagnosing HCC. The major organizations for liver studies, including the American Association for the Study of Liver Diseases, ${ }^{3}$ Asian Pacific Association for the Study of the Liver, ${ }^{10}$ European Association for the Study of the Liver, ${ }^{37}$ and Japanese Society of Hepatology, ${ }^{38}$ have each published diagnostic guidelines for HCC. The Asian Pacific Association for the Study of the Liver ${ }^{39}$ and Japanese Society of Hepatology $y^{38}$ guidelines recommend an algorithm that begins with tumor arterial enhancement. Tumor vascularity is a very important diagnostic factor for liver tumors.

\section{Hypervascular HCC}

Advanced HCC lesions usually exhibit hypervascularity. Therefore, the detection of typical HCC is almost equal to detection of hypervascular HCC. Contrast-enhanced US exhibits the same hypervascular HCC detection sensitivity as dynamic CT. ${ }^{19}$ Gd-EOB-DTPA MR imaging exhibits better detection sensitivity than dynamic CT or dynamic MRI with Gd-DTPA and sensitivity similar to that of superparamagnetic iron oxide-enhanced MR imaging and CT during arterial portography and hepatic angiography. ${ }^{12,22,23}$ However, contrast-enhanced US, dynamic CT, or dynamic MR imaging with Gd-DTPA is employed for HCC screening because the imaging quality and diagnostic ability of each modality might change depending on each institutional situation (eg, the specialties of radiologists and technologists and quality of the imaging unit). Table 1 shows the different studies in which the performance of dynamic-enhanced CT or MR imaging for $\mathrm{HCC}$ diagnosis was verified against histologic findings as a reference standard. The low sensitivity of dynamic-enhanced CT and MR imaging for diagnosing small HCC nodules could be explained by difficulties in detection and characterization of nodules with atypical enhancement. ${ }^{42-51}$ 
Table I Reported sensitivities and specificities of dynamicenhanced CT and MRI with extracellular contrast material in diagnosing hepatocellular carcinoma against histological findings as a standard of reference

\begin{tabular}{|c|c|c|c|c|}
\hline Reference & $\begin{array}{l}\text { Imaging } \\
\text { technique }\end{array}$ & $\begin{array}{l}\text { Mean } \\
\text { diameter } \\
(\mathrm{mm})\end{array}$ & $\begin{array}{l}\text { Sensitivity } \\
\text { (\%) }\end{array}$ & $\begin{array}{l}\text { Specificity } \\
(\%)\end{array}$ \\
\hline Lim et $\mathrm{al}^{42}$ & Dynamic CT & 19 & 71 & \\
\hline $\begin{array}{l}\text { Krinsky } \\
\text { et al }{ }^{43}\end{array}$ & Dynamic MRI & 18 & 55 & \\
\hline \multirow[t]{2}{*}{ Rode et $\mathrm{al}^{44}$} & Dynamic CT & $>8$ & 53.8 & \\
\hline & Dynamic MRI & $>8$ & 76.9 & \\
\hline Krinsky & Dynamic MRI & $>20$ & 100 & \\
\hline \multirow[t]{2}{*}{ et $\mathrm{al}{ }^{45}$} & & $10-20$ & 52 & \\
\hline & & $<10$ & 4 & \\
\hline $\begin{array}{l}\text { Brancatelli } \\
\text { et } \mathrm{al}^{46}\end{array}$ & Dynamic CT & & 60.8 & 52 \\
\hline \multirow[t]{2}{*}{ Valls et $\mathrm{a}^{47}$} & Dynamic CT & $>20$ & 93.6 & \\
\hline & & $<20$ & 61 & \\
\hline \multirow[t]{4}{*}{ Kim et $\mathrm{al}^{48}$} & Dynamic CT & $>10$ & 91.3 & 95.3 \\
\hline & & $<10$ & 29 & \\
\hline & Dynamic MRI & $>10$ & 90.2 & 97 \\
\hline & & $<10$ & 29 & \\
\hline Ronzoni et $\mathrm{a}^{49}$ & Dynamic CT & 17 & 77 & 75 \\
\hline Forner et $\mathrm{al}^{50}$ & Dynamic MRI & $<20$ & 61.7 & 96.6 \\
\hline Sofue et $\mathrm{a}^{51}$ & Dynamic CT & 21 & 92.9 & 93.7 \\
\hline
\end{tabular}

Abbreviations: CT, computed tomography; MRI, magnetic resonance imaging.

Enhancement of the arterial parenchyma due to an arterial-portal venous shunt might appear as a falsepositive lesion during dynamic MDCT investigations of HCC. Some HCC, which do not exhibit washout of contrast medium during the portal or equilibrium phase, may be difficult to diagnose. Sonazoid-enhanced US or Gd-EOB-DTPA-enhanced MR imaging is performed for the differential diagnosis of an arterial-portal shunt. Hypo-echo in the Kupffer phase of Sonazoidenhanced US or hypointensity in the hepatocyte phase of Gd-EOB-DTPA-enhanced MR imaging is diagnostic for HCC.

Shimizu et $\mathrm{a}^{52}$ demonstrated that a significant percentage of small early arterial-enhancing lesions in patients with cirrhosis or chronic hepatitis were not HCC. Of 104 small $(<20 \mathrm{~mm})$ arterial-enhancing lesions that were round or oval, only $28 \%$ were classified as HCC based on interval growth or a pathologic confirmation. More than half $(52 \%)$ of these lesions were considered to be definitely not HCC and $20 \%$ of the lesions, which featured a stable size and appearance, were considered likely pseudolesions. Moreover, in the study by Shimizu et al, $48 \%$ of the small HCCs were detected only in the arterial phase, and these nodules did not exhibit the typical washout pattern in the portal and delayed phases.

\section{Hypovascular HCC}

As stated above, it is crucial to detect lesion hypervascularity in order to make a diagnosis of $\mathrm{HCC}$, because this feature is among the most reliable characteristics of HCC. However, early or well differentiated $\mathrm{HCC}$ often exhibits hypovascularity. ${ }^{8,53}$ Several studies ${ }^{54-56}$ have demonstrated that $27 \%-34 \%$ of small HCCs are hypovascular. Therefore, the actual criteria, which are based only on a "typical" vascular enhancement pattern, should be reconsidered, especially for cases of HCCs that are $<20 \mathrm{~mm}$ in diameter. Dynamic MDCT and dynamic MR imaging with Gd-DTPA are not particularly useful for detecting hypovascular lesions, nor is superparamagnetic iron oxide-enhanced MR imaging because hypovascular HCC often contains Kupffer cells ${ }^{57}$ and thus takes up superparamagnetic iron oxide. Currently, there are no reports indicating the usefulness of enhanced US for detecting hypovascular HCC. Instead, Gd-EOB-DTPA-enhanced MR imaging was reported to be superior to other imaging modalities for detection of these lesions. ${ }^{22,23}$

Gd-EOB-DTPA-enhanced MR imaging can detect hypovascular and well differentiated HCC lesions. Using Gd-EOB-DTPA-enhanced MR imaging for chronic liver disease, we often encounter tiny hypovascular nodules $(<15 \mathrm{~mm}$ in diameter) that can only be detected as hypointense nodules on hepatocyte-phase images (Figure 2). ${ }^{58,59}$ The clinical management of these tiny hypovascular nodules is currently discussed because precancerous lesions may be present at a particular rate in hypovascular HCC. For example, hypovascular nodules exhibiting hypointensity on hepatocyte-phase Gd-EOB-DTPA-enhanced MR images can transition to $\mathrm{HCC}$ within 2 or 3 years at a rate of $11.1 \%$ or $15.9 \%$, respectively ${ }^{60}$ High intensity on T2-weighted images, fat deposition, a diameter of $10-15 \mathrm{~mm}$ at the time of detection, and hypointensity on the hepatocytephase Gd-EOB-DTPA-enhanced MR images are reported risk factors for carcinogenesis. ${ }^{58,61-63}$ Tiny hypovascular nodules with a doubling time of $<500$ days exhibit a high level of tumor hypervascularization during follow-up. ${ }^{63}$ Therefore, the recommended management for these tiny hypovascular nodules that are detected only on Gd-EOBDTPA-enhanced MR images is as follows: biopsy can be performed for lesions ranging from $10 \mathrm{~mm}$ to $15 \mathrm{~mm}$ in diameter, ${ }^{3,37}$ lesions $<10 \mathrm{~mm}$ in diameter should be followed up with dynamic MDCT, enhanced (Sonazoid) US, or Gd-EOB-DTPA-enhanced MR imaging, and biopsy can be performed when the lesion increases in size; imaging examinations are recommended every 3-6 months for 

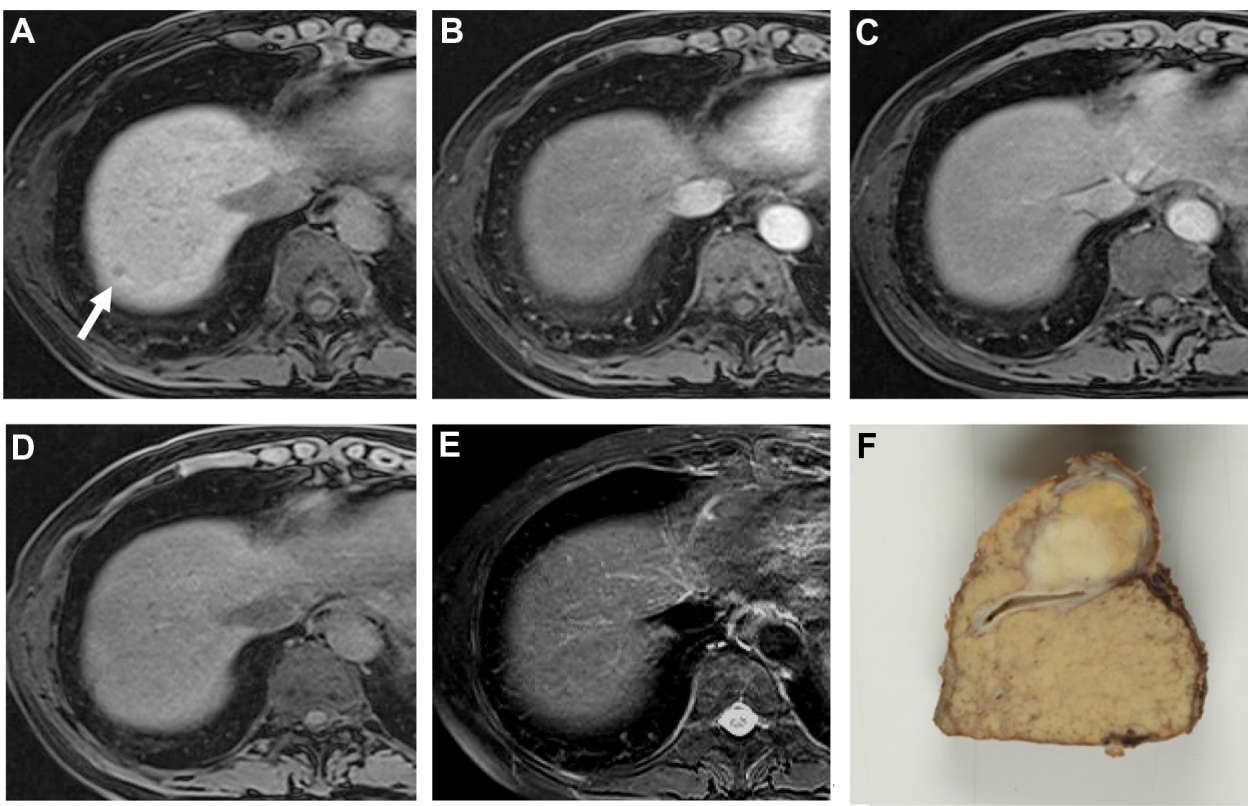

Figure 2 A small hepatocellular carcinoma in a 55-year-old man.

Notes: (A) A hepatobiliary phase image of Gd-EOB-DTPA-enhanced magnetic resonance imaging shows a small hypointense lesion in segment 8 (arrow). The lesion is not seen on other sequences, ie, (B) arterial phase, (C) portal phase, (D) precontrast TI-weighted image, and (E) a fat-suppressed T2-weighted image. (F) The lesion shows considerable enlargement on the follow-up image obtained one year after the first examination. Surgical specimens revealed a well differentiated hepatocellular carcinoma. Abbreviation: Gd-EOB-DTPA, gadolinium ethoxybenzyl diethylenetriamine pentaacetic acid.

lesions approximately $15 \mathrm{~mm}$ in diameter because lesions should be treated before reaching $20 \mathrm{~mm}$ in diameter; ${ }^{63}$ and a lesion can be treated when it exhibits hypervascularization on follow-up images.

\section{Infiltrating HCC}

Although infiltrating HCC accounts for $7 \%-13 \%$ of all HCC cases $^{64,65}$ and is thus not an uncommon HCC subtype, it remains poorly characterized in the literature. Infiltrating HCC exhibits a diffuse, permeating appearance on crosssectional imaging and is difficult to detect in settings such as the heterogeneous background of a cirrhotic liver; therefore, infiltrating $\mathrm{HCC}$ is often not diagnosed until it has progressed to an advanced stage. ${ }^{66}$ Specifically, infiltrating HCC often presents as predominantly hypointense on T1-weighted images and usually as homogeneous and mild to moderately hyperintense on T2-weighted images. ${ }^{65}$ On gadoliniumenhanced dynamic imaging, the majority of infiltrating HCC lesions appear as inhomogeneous areas of enhancement on arterial-phase images and as corresponding washout on more delayed phases of contrast enhancement. In addition, infiltrating HCC-associated portal vein thrombus commonly displays significant neovascularity or "arterialization" of the tumor thrombus. In fact, it is not uncommon for neovascularity of portal vein thrombus to be the only initial detectable imaging characteristic of an infiltrating HCC. ${ }^{65,67}$

\section{Staging}

A chest CT, bone scintigraphy, or PET/CT examination should be performed for a patient with risk factors for extrahepatic metastasis, such as the presence of portal vein tumor thrombus, an alpha-fetoprotein level $>200 \mathrm{ng} / \mathrm{mL}$, a PIVKA-II level $>300 \mathrm{mAU} / \mathrm{mL}$, a platelet count $>130 \times 10^{3} / \mu \mathrm{L}$, or age $<65$ years. Extrahepatic metastasis of HCC occurs rarely in new-onset cases $(1.0 \%-2.3 \%)^{68}$ but is found more frequently $(21 \%-24 \%)$ during the follow-up period after treatment. ${ }^{69} \mathrm{~A}$ brain $\mathrm{CT}$ or MR imaging can be performed if neurologic symptoms or lung metastasis are present.

\section{Disclosure}

The authors have no conflicts of interest to disclose with respect to this review paper.

\section{References}

1. Bosch FX, Ribes J, Díaz M, Cléries R. Primary liver cancer: worldwide incidence and trends. Gastroenterology. 2004;127 Suppl 1:S5-S16.

2. Degos F, Christidis C, Ganne-Carrie N, et al. Hepatitis C virus related cirrhosis: time to occurrence of hepatocellular carcinoma and death. Gut. 2000;47:131-136.

3. Bruix J, Sherman M. American Association for the Study of Liver Diseases: management of hepatocellular carcinoma: an update. Hepatology. 2011;53:1020-1022.

4. Burns PN, Wilson SR. Microbubble contrast for radiological imaging: 1. Principles. Ultrasound Q. 2006;22:5-13.

5. Bhayana D, Kim TK, Jang HJ, Burns PN, Wilson SR. Hypervascular liver masses on contrast-enhanced ultrasound: the importance of washout. AJR Am J Roentgenol. 2010;194:977-983. 
6. Yanagisawa K, Moriyasu F, Miyahara T, Yuki M, Iijima H. Phagocytosis of ultrasound contrast agent microbubbles by Kupffer cells. Ultrasound Med Biol. 2007;33:318-325.

7. Moriyasu F, Itoh K. Efficacy of perflubutane microbubble-enhanced ultrasound in the characterization and detection of focal liver lesions: phase 3 multicenter clinical trial. AJR Am J Roentgenol. 2009;193: $86-95$.

8. Kim TK, Lee KH, Khalili K, Jang HJ. Hepatocellular nodules in liver cirrhosis: contrast-enhanced ultrasound. Abdom Imaging. 2011;36:244-263.

9. Xu HX, Liu GJ, Lu MD, et al. Characterization of focal liver lesions using contrast-enhanced sonography with a low mechanical index mode and a sulfur hexafluoride-filled microbubble contrast agent. J Clin Ultrasound. 2006;34:261-272.

10. Xu HX, Liu GJ, Lu MD, et al. Characterization of small focal liver lesions using real-time contrast-enhanced sonography: diagnostic performance analysis in 200 patients. J Ultrasound Med. 2006;25:349-361.

11. Murakami T, Onishi H, Mikami K, et al. Determining the optimal timing for early arterial phase hepatic CT imaging by measuring abdominal aortic enhancement in variable contrast injection protocols. J Comput Assist Tomogr. 2006;630:206-211.

12. Noguchi Y, Murakami T, Kim T, et al. Detection of hepatocellular carcinoma: comparison of dynamic MR imaging with dynamic double arterial phase helical CT. AJR Am J Roentgenol. 2003;180 $455-460$.

13. Takahashi S, Murakami T, Takamura M, et al. Multi-detector row helical CT angiography of hepatic vessels: depiction with dual-arterial phase acquisition during single breath hold. Radiology. 2002;222:81-88.

14. Brooks RA. A quantitative theory of the Hounsfield unit and its application to dual energy scanning. $J$ Comput Assist Tomogr. 1977;1:487-493.

15. Nakayama Y, Awai K, Funama Y, et al. Abdominal CT with low tube voltage: preliminary observations about radiation dose, contrast enhancement, image quality, and noise. Radiology. 2005;237: 945-951.

16. Schindera ST, Nelson RC, Mukundan S Jr, et al. Hypervascular liver tumors: low tube voltage, high tube current multi-detector row CT for enhanced detection - phantom study. Radiology. 2008;246: $125-132$

17. Marin D, Nelson RC, Samei E, et al. Hypervascular liver tumors: low tube voltage, high tube current multidetector CT during later hepatic arterial phase for detection-initial clinical experience. Radiology. 2009;251:771-779.

18. Yeh BM, Shepherd JA, Wang ZJ, et al. Dual-energy and low-kVp CT in the abdomen. AJR Am J Roentgenol. 2009;193:47-54.

19. Imai Y, Murakami T, Yoshida S, et al. Superparamagnetic iron oxideenhanced MR images of hepatocellular carcinoma: correlation with histological grading. Hepatology. 2000;32:205-212.

20. Saito K, Kotake F, Ito N, et al. Gd-EOB-DTPA enhanced MRI for hepatocellular carcinoma: quantitative evaluation of tumor enhancement in hepatobiliary phase. Magn Reson Med Sci. 2005;4:1-9.

21. Tsuda N, Okada M, Murakami T. Potential of gadolinium-ethoxybenzyldiethylenetriamine pentaacetic acid (Gd-EOB-DTPA) for differential diagnosis of nonalcoholic steatohepatitis and fatty liver in rats using magnetic resonance imaging. Invest Radiol. 2007;42:242-247.

22. Kogita S, Imai Y, Okada M, et al. Gd-EOB-DTPA-enhanced magnetic resonance images of hepatocellular carcinoma: correlation with histological grading and portal blood flow. Eur Radiol. 2010;20 2405-2413.

23. Okada M, Imai Y, Kim T, et al. Comparison of enhancement patterns of histologically confirmed hepatocellular carcinoma between gadoxetateand ferucarbotran-enhanced magnetic resonance imaging. JMagn Reson Imaging. 2010;32:903-913.

24. Kagawa Y, Okada M, Kumano S, et al. Optimal scanning protocol of arterial dominant phase for hypervascular hepatocellular carcinoma with gadolinium-ethoxybenzyl-diethylenetriamine pentaacetic acidenhanced MR. J Magn Reson Imaging. 2011;33:864-872.
25. Hamm B, Staks T, Muhler A, et al. Phase I clinical evaluation of Gd-EOB-DTPA as a hepatobiliary MR contrast agent: safety, pharmacokinetics, and MR imaging. Radiology. 1995;195:785-792.

26. Bellin MF, Vasile M, Morel-Precetti S. Currently used non-specific extracellular MR contrast media. Eur Radiol. 2003;13:2688-2698.

27. Brasch RC. New directions in the development of MR imaging contrast media. Radiology. 1992;183:1-11.

28. Sano K, Ichikawa T, Motosugi U, et al. Imaging study of early hepatocellular carcinoma: usefulness of gadoxetic acid-enhanced MR imaging. Radiology. 2011;261:834-844.

29. Narita M, Hatano E, Arizono S, et al. Expression of OATP1B3 determines uptake of Gd-EOB-DTPA in hepatocellular carcinoma. J Gastroenterol. 2009;44:793-798.

30. Tsuboyama T, Onishi H, Kim T, et al. Hepatocellular carcinoma: hepatocyte-selective enhancement at gadoxetic acid-enhanced MR imaging - correlation with expression of sinusoidal and canalicular transporters and bile accumulation. Radiology. 2010;255:824-833.

31. Kitao A, Zen Y, Matsui O, et al. Hepatocellular carcinoma: signal intensity at gadoxetic acid-enhanced MR imaging - correlation with molecular transporters and histopathologic features. Radiology. 2010;256:817-826.

32. Di Martino M, Marin D, Guerrisi A, et al. Intraindividual comparison of gadoxetate disodium-enhanced MR imaging and 64-section multidetector $\mathrm{CT}$ in the detection of hepatocellular carcinoma in patients with cirrhosis. Radiology. 2010;256:806-816.

33. Park G, Kim YK, Kim CS, Yu HC, Hwang SB. Diagnostic efficacy of gadoxetic acid-enhanced MRI in the detection of hepatocellular carcinomas: comparison with gadopentetate dimeglumine. Br J Radiol. 2010;83:1010-1016

34. Onishi H, Kim T, Imai Y, et al. Hypervascular hepatocellular carcinomas: detection with gadoxetate disodium-enhanced MR imaging and multiphasic multidetector CT. Eur Radiol. 2012;22: 845-854.

35. Tsurusaki M, Okada M, Kuroda H, Matsuki M, Ishii K, Murakami T. Clinical application of $18 \mathrm{~F}$-fluorodeoxyglucose positron emission tomography for assessment and evaluation after therapy for malignant hepatic tumor. $J$ Gastroenterol. 2014;49:46-56.

36. Sugiyama M, Sakahara H, Torizuka T, et al. 18F-FDG PET in the detection of extrahepatic metastases from hepatocellular carcinoma. J Gastroenterol. 2004;39:961-968.

37. European Association for the Study of the Liver, European Organization for Research and Treatment of Cancer. EASL-EORTC clinical practice guidelines: management of hepatocellular carcinoma. $J$ Hepatol. 2012;56:908-943.

38. Kudo M, Izumi N, Kokudo N, et al. HCC Expert Panel of Japan Society of Hepatology. Management of hepatocellular carcinoma in Japan: consensus-based clinical practice guidelines proposed by the Japan Society of Hepatology (Japanese Society of Hepatology) 2010 updated version. Dig Dis. 2011;29:339-364.

39. Omata M, Lesmana LA, Tateishi R, et al. Asian Pacific Association for the Study of the Liver consensus recommendations on hepatocellular carcinoma. Hepatol Int. 2010;4:439-474.

40. Matsui O, Kadoya M, Kameyama T, et al. Benign and malignant nodules in cirrhotic livers: distinction based on blood supply. Radiology. 1991;178:493-497.

41. Hayashi M, Matsui O, Ueda K, et al. Correlation between the blood supply and grade of malignancy of hepatocellular nodules associated with liver cirrhosis: evaluation by CT during intraarterial injection of contrast medium. AJR Am J Roentgenol. 1999;172: 969-976.

42. Lim JH, Kim CK, Lee WJ, et al. Detection of hepatocellular carcinomas and dysplastic nodules in cirrhotic livers: accuracy of helical CT in transplant patients. AJR Am J Roentgenol. 2000;175:693-698.

43. Krinsky GA, Lee VS, Theise ND, et al. Hepatocellular carcinoma and dysplastic nodules in patients with cirrhosis: prospective diagnosis with MR imaging and explantation correlation. Radiology. 2001;219:445-454. 
44. Rode A, Bancel B, Douek P, et al. Small nodule detection in cirrhotic livers: evaluation with US, spiral CT, and MRI and correlation with pathologic examination of explanted liver. J Comput Assist Tomogr. 2001;25:327-336.

45. Krinsky GA, Lee VS, Theise ND, et al. Transplantation for hepatocellular carcinoma and cirrhosis: sensitivity of magnetic resonance imaging. Liver Transpl. 2002;8:1156-1164.

46. Brancatelli G, Baron RL, Peterson MS, Marsh W. Helical CT screening for hepatocellular carcinoma in patients with cirrhosis: frequency and causes of false-positive interpretation. AJR Am J Roentgenol. 2003;180: 1007-1014.

47. Valls C, Cos M, Figueras J, et al. Pretransplantation diagnosis and staging of hepatocellular carcinoma in patients with cirrhosis: value of dual-phase helical CT. AJR Am J Roentgenol. 2004;182:1011-1017.

48. Kim SH, Choi D, Lim JH, et al. Ferucarbotran-enhanced MRI versus triple-phase MDCT for the preoperative detection of hepatocellular carcinoma. AJR Am J Roentgenol. 2005;184:1069-1076.

49. Ronzoni A, Artioli D, Scardina R, et al. Role of MDCT in the diagnosis of hepatocellular carcinoma in patients with cirrhosis undergoing orthotopic liver transplantation. AJR Am J Roentgenol. 2007;189:792-798.

50. Forner A, Vilana R, Ayuso C, et al. Diagnosis of hepatic nodules $20 \mathrm{~mm}$ or smaller in cirrhosis: prospective validation of the noninvasive diagnostic criteria for hepatocellular carcinoma. Hepatology. 2008;47:97-104.

51. Sofue K, Tsurusaki M, Kawasaki R, Fujii M, Sugimura K. Evaluation of hypervascular hepatocellular carcinoma in cirrhotic liver: comparison of different concentrations of contrast material with multi-detector row helical CT - a prospective randomized study. Eur J Radiol. 2011;80:e237-e242.

52. Shimizu A, Ito K, Koike S, Fujita T, Shimizu K, Matsunaga N. Cirrhosis or chronic hepatitis: evaluation of small $(\leq 2-\mathrm{cm})$ early enhancing hepatic lesions with serial contrast-enhanced dynamic MR imaging. Radiology. 2003;226:550-555.

53. Jang HJ, Kim TK, Burns PN, Wilson SR. Enhancement patterns of hepatocellular carcinoma at contrast-enhanced US: comparison with histologic differentiation. Radiology. 2007;244:898-906.

54. Heilmaier C, Lutz AM, Bolog N, Weishaupt D, Seifert B, Willmann JK. Focal liver lesions: detection and characterization at doublecontrast liver MR Imaging with ferucarbotran and gadobutrol versus single-contrast liver MR imaging. Radiology. 2009;253:724-733.

55. Hayashi M, Matsui O, Ueda K, Kawamori Y, Gabata T, Kadoya M. Progression to hypervascular hepatocellular carcinoma: correlation with intranodular blood supply evaluated with CT during intraarterial injection of contrast material. Radiology. 2002;225:143-149.

56. Bolondi L, Gaiani S, Celli N, et al. Characterization of small nodules in cirrhosis by assessment of vascularity: the problem of hypovascular hepatocellular carcinoma. Hepatology. 2005;42:27-34.
57. Tanaka M, Nakashima O, Wada Y, Kage M, Kojiro M. Pathomorphological study of Kupffer cells in hepatocellular carcinoma and hyperplastic nodular lesions in the liver. Hepatology. 1996;24: 807-812.

58. Kumada T, Toyoda H, Tada T, et al. Evolution of hypointense hepatocellular nodules observed only in the hepatobiliary phase of gadoxetate disodium-enhanced MRI. AJR Am J Roentgenol. 2011;197:58-63.

59. Motosugi U, Ichikawa T, Sano K, et al. Outcome of hypovascular hepatic nodules revealing no gadoxetic acid uptake in patients with chronic liver disease. J Magn Reson Imaging. 2011;34:88-94.

60. Akai H, Matsuda I, Kiryu S, et al. Fate of hypointense lesions on Gd-EOB-DTPA-enhanced magnetic resonance imaging. Eur J Radiol. 2012;81:2973-2977.

61. Kobayashi S, Matsui O, Gabata T, et al. Gadolinium ethoxybenzyl diethylenetriamine pentaacetic acid-enhanced magnetic resonance imaging findings of borderline lesions at high risk for progression to hypervascular classic hepatocellular carcinoma. J Comput Assist Tomogr. 2011;35:181-186.

62. Yu JS, Chung JJ, Kim JH, Kim KW. Fat-containing nodules in the cirrhotic liver: chemical shift MRI features and clinical implications. AJR Am J Roentgenol. 2007;188:1009-1016.

63. Hyodo T, Murakami T, Imai Y, et al. Hypovascular nodules in chronic liver disease: risk factors for developing hypervascular hepatocellular carcinoma. Radiology. 2013;266:480-490.

64. Trevisani F, Caraceni P, Bernardi M, et al. Gross pathologic types of hepatocellular carcinoma in Italian patients. Relationship with demographic, environmental, and clinical factors. Cancer. 1993;72: 1557-1563.

65. Kanematsu M, Semelka RC, Leonardou P, et al. Hepatocellular carcinoma of diffuse type: MR imaging findings and clinical manifestations. J Magn Reson Imaging. 2003;18:189-195.

66. Mehta N, Fidelman N, Sarkar M, Yao FY. Factors associated with outcomes and response to therapy in patients with infiltrative hepatocellular carcinoma. Clin Gastroenterol Hepatol. 2013;11: $572-578$.

67. Kim YK, Han YM, Kim CS. Comparison of diffuse hepatocellular carcinoma and intrahepatic cholangiocarcinoma using sequentially acquired gadolinium-enhanced and Resovist-enhanced MRI. Eur $J$ Radiol. 2009;70:94-100.

68. Uchino K, Tateishi R, Shiina S, et al. Hepatocellular carcinoma with extrahepatic metastasis: clinical features and prognostic factors. Cancer. 2011;117:4475-4483.

69. Senthilnathan S, Memon K, Lewandowski RJ, et al. Extrahepatic metastases occur in a minority of hepatocellular carcinoma patients treated with locoregional therapies: analyzing patterns of progression in 285 patients. Hepatology. 2012;55:1432-1442.

\section{Publish your work in this journal}

The Journal of Hepatocellular Carcinoma is an international, peerreviewed, open access journal that offers a platform for the dissemination and study of clinical, translational and basic research findings in this rapidly developing field. Development in areas including, but not limited to, epidemiology, vaccination, hepatitis therapy, pathology and

\section{Dovepress}

molecular tumor classification and prognostication are all considered for publication. The manuscript management system is completely online and includes a very quick and fair peer-review system, which is all easy to use. Visit http://www.dovepress.com/testimonialsphp to read real quotes from published authors. 